\title{
A REVIEW OF RECENT ULTRAVIOLET OBSERVATIONS OF CLOSE BINARIES
}

FROM SPACE

\section{Yoji Kondo}

Laboratory for Astronomy and Solar Physics, NASA/Goddard Space Flight Center, Greenbelt, MD 20771, U.S.A.

The reason for presenting this review at this Joint Meeting, in spite of the fact that there was, a few days ago, a special Joint Discussion on recent UV observations is partially that somehow the general subject of close binaries was overlooked at the Joint Discussion, although unique close binaries, such as cataclysmic binaries and $\mathrm{X}$-ray binaries, were included. There exist a large number of vigorous research projects being carried out with UV telescopes in the field of close binaries. I shall concentrate on IUE results, although other satellite and balloon-borne telescopes have continued to produce interesting UV observations of close binaries.

There are a number of active IUE programs on this theme. In the time allocated to this review I shall only be able to present highlights of selected programs in the hope of giving a glimpse of what is going on. The programs on close binaries from the first year of IUE operations include those by P. Conti, M. Hack, J.B. Hutchings, R.H. Koch, Y. Kondo and G.E. McCluskey, M. Plavec, and J. Sahade. In the second year, additional astronomers are also observing close binaries with the IUE.

Under somewhat expanded interpretation of the theme of this Joint Meeting, i.e., stellar activity, I shall report mainly on the mass flow within and out of close binaries. With the advent of large-capacity computers the complex problem of mass flow could be analysed from the view point of hydrodynamics. We are now witnessing the first steps taken in this direction. However, in order for us to develop a viable hydrodynamic treatment of the problem, we critically need information on the boundary conditions and other physical parameters on the gas flow, e.g., the directions of the flow, the temperatures and densities of the plasma, etc. Hence, these results under discussion are quite important in furthering our understanding of the physical conditions and evolution of close binaries.

(A) Conti reports that the mass loss rates from single stars and close binaries of 0 types do not differ materially from each other. 
(B) Hack observed the symbiotic star $\mathrm{CH}$ Cyg; other close binaries, such as $\beta$ Lyr and $\varepsilon$ Aur, were also observed in her program. CH Cyg is normally an M6 III object. Its active phase recurs every several years; it was observed in 1978 April and July during its active phase. Resonance lines of neutral and once-ionized metals were observed in emission typically at wavelengths longward of $1700 \AA$. The continuum level, estimated from ground-based data, indicated a temperature of $10^{4} \mathrm{~K}$ during the outburst. The continuum between 1250 and $1700 \AA$ was flat; 0 I at $1302 \AA$ was observed as a strong emission feature. We are still unable to decide between the two existing hypotheses regarding the nature of symbiotic objects based on the current UV results. The hypotheses are: (a) a late type giant is accompanied by a hot subdwarf that undergoes outbursts, and (b) a cool star is surrounded by an extended thick corona heated by shock waves.

(c) Hutchings observed a number of hot binaries, including v861 Sco. Its earlier identification with an X-ray source has.recently been refuted, but it is an interesting binary in its own right. (It was also observed by Kondo and McCluskey, among others.) Orbital motion was reflected in weak and medium lines. The variation in resonance lines near phase 0.5 was similar to that observed in $\mathrm{HD} 77581=\mathrm{Vela} \mathrm{X}-1$. Lower ionization lines showed Doppler-shifting corresponding to one third of the terminal velocity seen in strong $P$ Cygni absorption lines. The continuum has the same slope throughout the binary phases, even at eclipse. The UV light curve, generated from a number of IUE spectra, had deeper minima than in the visible light.

(D) Plavec and Koch, in addition to other projects, undertook to collaborate in an effort to look for close binaries surrounded by hot plasma such as that observed in $B$ Lyr. The presence of such hot plasma was envisaged from earlier OAO-2 UV photometric light curves (Kondo, McCluskey and Houck 1971; Kondo, McCluskey and Eaton 1976), which showed deepening secondary minimum in the shorter wavelengths in the UV. A series of papers based on Copermicus UV spectra obtained and analysed by an international team of observers (Hack, Hutchings, Kondo, McCluskey, Plavec and variously involving Polidan and Tulloch) showed conclusively that $\beta$ Lyr was surrounded by hot plasma characteristically seen in multitudinous emission lines of multi-ionized elements. The emission lines are resonance transitions: These observations indicated that the hot plasma probably arose from the accretion process and that the emission lines are caused by collisional processes.

Plavec and Koch were handsomely rewarded in their search by the discovery of five additional binaries that exhibited " $\beta$ Lyr" like UV spectra. The objects so identified are RX Cas, SX Cas, W Cru, V367 Cyg and W Ser. In the IUE low resolution $(6-10 \AA)$ mode, in which these binaries were observed, the spectra showed: (a) apparent continuum hotter for the spectral type (probably to be resolved into multitudinous emission lines at a higher resolution), (b) strong resonance emission lines of $\mathrm{N} \mathrm{V,C} \mathrm{IV,}$ C III, Si IV, Si III, Si II, Fe III, Al III, Ni II, etc. The flux contained in these strong emission lines are $10^{3}$ to $10^{4}$ times greater than that from ordinary chromospheres. 
(E) Kondo and McCluskey observed systematically a number of close binaries that appeared suitable for study of mass flow. The binaries observed at high resolution included $\mu \mathrm{Sgr}$, U Sgr, AO Cas, B Lyr, 32 Cyg and $\mathrm{HD} 77581=\mathrm{Vela} X-1$. One of the interesting results was the observation of gas streaming in U Cep. The temperature and the density of the plasma in the gas stream were such that the signature of the stream was most clearly seen in the Fe II resonance line at $2599 \AA$ and the $\mathrm{Mg}$ II resonance doublet at 2795 and $2802 \AA$. The signature of the gas stream was observed as shortward and longward shifted absorption as a function of phase; the excess absorption due to the gas stream was superimposed on the underlying photospheric absorption but was unmistakably present. According to Batten (1974), this binary consists of a B7 V and a G8 III objects. At the time of the IUE observations in 1978 and 1979, the mass appeared to leave the G star's hemisphere facing the B star, goes around the forward hemisphere of the B star, and left the binary system in the direction of the viewing angle for phases $0.15-0.20$. A portion of the gas leaving the $G$ star was probably being accreted to the $B$ star, judging from the likely presence of hot spots previously reported by a number of investigators. It is also conceivable that a gas stream at a different temperature - hence unobserved in the current data - was present and had a different flow pattern, perhaps even forming a disk around the $B$ star as inferred by other observers. The present results were obtained during U Cep's quiescent period based on private communication from Olson.

The fact that the matter was leaving the binary system indicates that there existed a source of energy propelling the stream, in addition to the simple conversion of the gravitational potential to kinetic energy. We speculate that the non-linear (g-mode) oscillation in the G star gives rise to local heating and mass ejection in a manner envisaged by Wolff and Kondo (1978) and followed up in theoretical computations by Modisette and Kondo (1979).

(F) Sahade has obtained spectra of the symbiotic objects AR Pav and 17 Lep; he is in the process of analysing the data.

\section{REFERENCES}

Batten, A.H.: 1974, Publ. Dominion Astrophys. Obs. 14, 191. Kondo, Y., McCluskey, G.E., and Eaton, J.A.: 1976, Astrophys. Space Sci. Kondo, Y., MCCluskey, G.E., and Houck, T.E.: 1971, in A.D. Code (ed.), Scientific Resuits from OAO-2, NASA SP-310, Washington, p. 485.

Modisette, J.L. and Kondo, Y.: 1979, Astrophys. J., submitted. Wolff, C.L. and Kondo, Y.: 1978, Astrophys. J. 219, 605. 